\title{
A Survey on QoE-Oriented VR Video Streaming: Some Research Issues and Challenges
}

\author{
Jinjia Ruan (1) and Dongliang Xie * \\ State Key Laboratory of Networking and Switching Technology, Beijing University of Posts and \\ Telecommunications, Beijing 100876, China; jinjia_ruan@bupt.edu.cn \\ * Correspondence: xiedl@bupt.edu.cn
}

Citation: Ruan, J.; Xie, D. A Survey on QoE-Oriented VR Video Streaming: Some Research Issues and Challenges. Electronics 2021, 10, 2155. https://doi.org/10.3390/electronics 10172155

Academic Editor: Stefanos Kollias

Received: 29 July 2021

Accepted: 31 August 2021

Published: 3 September 2021

Publisher's Note: MDPI stays neutral with regard to jurisdictional claims in published maps and institutional affiliations.

Copyright: (c) 2021 by the authors. Licensee MDPI, Basel, Switzerland. This article is an open access article distributed under the terms and conditions of the Creative Commons Attribution (CC BY) license (https:// creativecommons.org/licenses/by/ $4.0 /)$.

\begin{abstract}
With the advent of the information age, VR video streaming services have emerged in large numbers in scenarios such as immersive entertainment, smart education, and the Internet of Vehicles. People are also demanding an increasing number of virtual-reality (VR) services, and service providers must ensure a good user experience. Therefore, the quality of the VR user's experience is receiving increasing attention from academia and industry. The review in this paper focuses on a comprehensive summary of the current state of quality-of-experience (QoE) technologies applied to VR video streaming. First, we review the main influencing factors of QoE and VR video streaming. Second, the user QoE for VR evaluation is discussed. Third, the modeling of QoE for VR video streaming, the QoE-oriented VR optimization problem, and enabling techniques of machine learning for VR video streaming improvement are summarized. Lastly, we present current challenges and possible future research directions.
\end{abstract}

Keywords: VR adaptive streaming; QoE; video quality assessment; machine learning

\section{Introduction}

With the development of Internet technology, network data traffic has shown an explosive growth trend. The most notable is the growth of video traffic, which brings huge challenges to current bearer networks. The popularization of virtual-reality (VR) applications has especially presented higher requirements for network quality and performance [1,2]. Since VR applications are dedicated to bringing immersive experiences, the perceived experience for user services is particularly important, where the perceived quality of user experience (QoE) for VR end users' services is a very important metric [3]. For the QoE of VR users, it is of great importance to understand the expectations and experiences of users for the development of existing services and the improvement of future services. Therefore, academic and industrial communities in the multimedia field show special interest in this area.

It is widely recognized that QoE is a multidisciplinary indicator that is influenced by a variety of factors from different fields. However, it can be very difficult to explicitly address the impact of all factors when they are taken into account. Most current studies on QoE evaluation are based on direct feedback from users to obtain subjective evaluations. Most subjective evaluation methods were developed for traditional video, are costly to collect user feedback, and data collection is limited by controlled environments. A traditional objective evaluation method of video quality is usually directly compared with the original video to arrive at the evaluation, which includes peak signal-to-noise ratio (PSNR) [4], structural similarity image metric (SSIM) [5], and the video-quality model (VQM) [6].

Although existing works studied the impact of user QoE on video services, most studies [7-14] only mention QoE in traditional video streaming applications. Since the interactive and immersive nature of VR video makes it vastly different from traditional flat image video, it makes traditional 2D QoE metrics not well-suited for the evaluation and 
optimization of existing VR video streams. Research on QoE related work for VR video streaming services has been gradually carried out over recent years.

\subsection{Survey Novelty and Contributions}

This survey is the first to discuss the current status of the impact and improvement of QoE on VR video streaming technology, and challenges and prospects for future development. Possible solutions for QoE assessment and optimization for VR users are critical to the success of VR service development. This survey focuses on the main trends in the application of QOE evaluation in VR video streaming optimization. Unlike previous studies that only briefly introduce the impact of QoE on VR, this paper focuses on a comprehensive survey of the main influencing factors of QoE for VR video streaming, evaluation methods, existing test platforms, and improvements of machine learning for QoE optimization. The main contributions of this paper are summarized as follows:

- An overview of QoE and VR video streaming is provided, and the benefits of QoE evaluation for the development of VR service applications are illustrated;

- Subjective and objective ways of evaluating VR QoE, and VR-related test and evaluation platforms are discussed;

- An evaluation model for QoE based on VR video streams is investigated, the optimization of VR systems is described as a QoE optimization option, and machine learning is considered an active way to support the QoE optimization of VR video streaming (machine learning is considered to be an active way to support QoE optimization of VR video streaming);

- For the enhancement of VR user experience, the challenges facing VR video streaming QoE and future research directions are described.

\subsection{Survey Structure}

The paper is organized as follows:

- Section 2: overview of the basic background knowledge on the main QoE influencing factors and VR video streaming;

- Section 3: description of QoE approach evaluation and testbed in targeting VR video applications;

- Section 4: constructed QoE models are surveyed on the basis of VR video streams, and QoE-oriented optimization problems for VR video streams; research on machinelearning methods for use in QoE optimization is examined;

- Section 5: recent findings are combined to provide an outlook on future research challenges and trends;

- Section 6: main points of this survey are summarized.

The roadmap of our approach is shown in Figure 1. Our work summarizes the QoE influencing factors of multiple dimensions in the frequency service, and uses the QoE evaluation model to evaluate whether the current user experience needs are met to guide the QoE optimization strategy, and then effectively improve the QoE. In the research work of QoE optimization, the selection of QoE influencing factors and the measurement and evaluation of QoE are common parts. QoE modeling and optimization are carried out on the basis of the former research output. 
A Survey on QoE-oriented VR Video Streaming

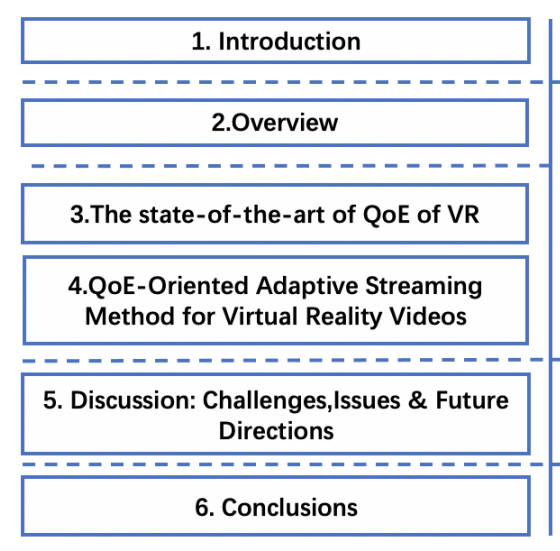

Figure 1. Paper structure.
Introduction of the subject and summary of the main contributions

Comprehension of what is QoE: influence factors and VR Streaming System

Review of state-of-the-art QoE-based on VR Video methods: Quality Assessment, QoE evaluation test platform,VR QoE Mode , optimization for virtual reality of existing work

Analysis of open challenges and future research opportunities.

Concluding remarks

\section{Overview}

In this section, the main QoE influencing factors are described, and the VR video streaming technology is introduced.

\subsection{QoE-Influencing Factors}

A study [15] considers QoE to be a research topic covering the multiple fields of computer science, social psychology, cognitive science, and economics. The study of QoE-influencing factors is the cornerstone of all QoE studies, which is difficult to directly obtain from some obvious factors, so the investigation of QoE-influencing factors needs to be comprehensively discussed. The white paper [16] on the definition of quality of experience defines influencing factors for QoE as "any characteristic of a user, system, service, application or environment whose actual state or setting may impact the user's quality of experience". According to ITU [17], the factors influencing QoE can include: the type and characteristics of the application or service, the environment in which it is used, the user's expectations and their fulfilment, cultural background, socioeconomic issues, psychological conditions, emotional state, and other factors, which may continue to grow as research progresses. From the above perspectives, we classify the influencing factors of QoE into the following categories, which include the influencing factors of the system's own properties on the experience (system factors), the influencing factors of the various external environments in which it is located (context factors), and the factors of the human's physiological and psychological perceptions of the experience (human factors), i.e,. System IF, Context IF, Human IF, shown in Table 1.

\subsubsection{System Factors}

A white paper published by Qualinet [16] indicates that the quality that an application or service technically produces is determined by system factors. QoE in video-delivery services may be affected by variations in the perceived quality of the content. Blockiness, blur, and other issues that may arise from different types of compression algorithms can lead to an unsatisfactory user experience. Meanwhile, system factors such as network QoS parameters [18] and media configurations [19] are also considered to hugely impact QoE. Dobrian et al. [18] argue that the percentage of buffering time in the total session time directly affects the QoE of the user, and the longer the buffering time is, the worse the QoE performance. A study [20] investigated other QoS parameters in the system (buffering event rate, buffering time, average bit rate) and showed great correlation with QoE.

In addition to this, studies [21] have argued that different forms of viewing content (e.g., competitions, conferences) lead to different viewing patterns, and that the user's QoE is influenced by the video content, and the user experiences different perceptual quality. The literature [22] shows that, with a given bit rate, genres that usually contain little motion 
are compared to genres that contain high-speed motion (action movies) to obtain a higher perceptual quality.

In summary, system factors for QoE can include network-layer (e.g., latency, throughput, packet loss, buffering event rate, buffering time, average bit rate, bandwidth), application-layer (e.g., resolution, frame rate), and service-layer (e.g., type of content viewed, application level, viewing mode) parameters.

\subsubsection{Context Factors}

Han et al. [23] argued that the user's QoE is influenced by some external factors that act on the surrounding environment, and when the user feels relaxed, this better impacts the user's quality of experience. Meanwhile, the location of the seat, viewing distance and height, lighting conditions [24], and possible disturbances [25] such as incoming calls or SMS alerts and some other physical-environment aspects may affect the user's experience. Martinez et al. considered some economic contextual situations, such as the cost of subscription type, among the factors influencing QoE. K.Yamori et al. [26] found that the user's payment for content impacts user experience. People usually show a higher experience tolerance for content with lower payment prices. A study [27-29] found that adding factors such as user expectations and budget, and quality pricing contributed to the accuracy of the user perception model.

In summary, environmental factors of QoE can include physical environmental (e.g., lighting, sound, location) and economic (e.g., desired price, budget) factors.

\subsubsection{Human Factors}

Both human physiological and psychological factors usually significantly impact QoE.

- Physiological factors

Most studies found that the user's physiological characteristics play a key role in in user QoE, with visual perception being of particular interest. Laghari et al. [30] analyzed a variety of factors in the human body itself (e.g., gender, age, etc.) to find the main influencing factors that may affect user perception quality. Most of these factors have been studied and modeled, but how an individual's physiological characteristics affect QoE is equally important. Owsley et al. [31] demonstrated that factors such as visual acuity and loss of contrast sensitivity due to aging can affect visibility (and annoyance) in visual impairment. However, they are hardly included in the QoE model. M.S. El-Nasr et al. [32] found that physiological deficit disorders of user vision directly impact the user experience. Colorblind users have a different perceptual experience, while stereoblindness can also hugely impact it when faced with an immersive visual experience. In addition, human auditory characteristics can impact the QoE of the medium. Saleme et al. [33] studied $360^{\circ}$ mulsemedia, an emerging VR application, with the aim of uncovering the physiological factors that may impact the experience. Unlike other factors, they introduced a specific factor of odor sensitivity. The study also found that women showed higher sensitivity when considering multisensory situations. P. Orero et al. [34] argue that the physiological situation can vary greatly in the assessment of the QoE in different situations due to the many different characteristics of the individual, minimizing the consideration of this aspect.

- Psychological factors

The user's psychological state is likely to play a large role in the level of satisfaction with the user experience. Some of the existing literature [35-40] indicated that personal psychological factors influence QoE in various ways, and Wechsung et al. [35] indicate that more variable factors, such as motivation, attention level, or user's mood, i.e., affective factors, also play an important role in dealing with QoE influencing factors. Another study [36], on the other hand, found that the effect of emotion and multimedia experience is reciprocal. A good experience leads to good emotions, which are more likely to produce a good experience. The authors of [37-39] found that interest is the 
influencing factor that plays a decisive role in QoE as an influencing factor of emotion, and that interest may be triggered by some content that may impact the perception of QoE. In [40], the authors experimentally found that, when people watch content in which they are interested, the effect of the video quality is ignored. Regarding video quality, interest is positively correlated with QoE. In addition, the authors in [41] argued that some other factors, such as education or occupation background, can also impact the QoE of users. In conclusion, the influence of personal psychological factors on perceived quality is complex and closely related.

In summary, human-level influencing factors of QoE are more subjective factors, mostly obtained through the users' active perception. They mainly include basic user profiles (e.g., age, gender, education level), physical state (e.g., vision, hearing, smell), physical and mental states (e.g., user preferences, emotions), background (e.g., educational and occupational background), and hobbies. These influencing factors are more complex and variable than objective factors.

Table 1. The influencing factors of QoE.

\begin{tabular}{|c|c|c|}
\hline QoE IFs & Type & Examples/References \\
\hline $\begin{array}{l}\text { System } \\
\text { Factors }\end{array}$ & Objective & $\begin{array}{l}\text { Network layer parameters (e.g., latency, throughput, packet loss, } \\
\text { buffering event rate, buffering time, average bit rate, } \\
\text { bandwidth [18]), application layer parameters (e.g., resolution, } \\
\text { frame rate [19]), service layer parameters (e.g., type of content } \\
\text { viewed, application level, viewing mode }[18,22] \text { ) }\end{array}$ \\
\hline $\begin{array}{l}\text { Context } \\
\text { Factors }\end{array}$ & Objective & $\begin{array}{l}\text { Physical environment factors (e.g., lighting, sound, location }[23,25] \text { ), } \\
\text { the Economic factors (e.g., desired price, budget [26-29]). }\end{array}$ \\
\hline $\begin{array}{l}\text { Physiological } \\
\text { Factors }\end{array}$ & Sbjective & $\begin{array}{l}\text { Basic user profile (e.g., age, gender, education level }[30,34]) \text {, the } \\
\text { physical state (e.g., vision, hearing, sense of smell }[31-33])\end{array}$ \\
\hline $\begin{array}{l}\text { Psychological } \\
\text { Factors }\end{array}$ & Sbjective & $\begin{array}{l}\text { physical/Mental status (e.g., user preferences, mood }[35,35]) \text {, } \\
\text { background (e.g., educational background, occupational } \\
\text { background [41]), hobbies/interests [37-40] }\end{array}$ \\
\hline
\end{tabular}

\subsection{VR Streaming System}

At this stage, most VR video services are segmented on the basis of adaptive streamingdelivery methods. Therefore, most VR video streaming systems are full-view streaming (viewport-independent), viewport-based streaming, and tile-based streaming. Next, we discuss representative streaming scenarios. These are summarized in Table 2.

Table 2. The influencing factors of QoE.

\begin{tabular}{|c|c|c|c|}
\hline & Full View Streaming & $\begin{array}{l}\text { Viewport-Based } \\
\text { Streaming }\end{array}$ & Tile-Based Streaming \\
\hline 360 video features & $\begin{array}{c}\text { Whole data frame in } \\
\text { same quality }\end{array}$ & $\begin{array}{c}\text { Viewport area in high } \\
\text { quality }\end{array}$ & $\begin{array}{l}\text { The tiles in same or } \\
\text { different quality }\end{array}$ \\
\hline Projection & CMP, ERP & $\begin{array}{c}\text { TSP, Pyramid, Offset } \\
\text { cubemap }\end{array}$ & $\begin{array}{c}\text { CMP, ERP, TSP, Pyramid, } \\
\text { Offset cubemap }\end{array}$ \\
\hline Encoding pressure & High & Medium & Low \\
\hline Cache pressure & High & Medium & Medium \\
\hline Bandwidth pressure & High & Medium & Medium \\
\hline $\begin{array}{l}\text { Adaptive influencing } \\
\text { factors }\end{array}$ & Network & $\begin{array}{l}\text { Network/Viewport, } \\
\text { Viewport size }\end{array}$ & $\begin{array}{l}\text { Network/Viewport, } \\
\text { Viewport/Tiles size }\end{array}$ \\
\hline
\end{tabular}

\subsubsection{Full-View Streaming}

Full-view streaming is the most straightforward available solution for streaming $360^{\circ}$ content. It delivers the entire frame in the same quality as that of a traditional streaming solution; $360^{\circ}$ video content is projected and encoded in ERP or CMP and then delivered directly to the client without the need to obtain additional HMD information. The client is also similar to the bit rate adaptation of traditional video, and continues the request for the clip on the basis of the current network state. Afzal et al. [42] conducted experiments 
on a large number of $360^{\circ}$ YouTube videos and did not consider the viewing direction to acquire only full frames. The results show that the bit rate of $360^{\circ}$ videos is about 6 times higher than that of regular videos. However, their encoding efficiency is greatly limited by the need to encode the entire frame compared to the viewport-related streaming media. In addition, they consume much bandwidth and many resources that are not decoded by the viewing area.

\subsubsection{Viewport-Based Streaming}

Viewport-based streaming is based on the selective transmission of viewports that people watch, providing high-quality transmission of the part that the user watches and low-quality transmission of the rest. On the client side, the streaming endpoint device detects the user's head movements and receives only certain desired video frame areas. The client reduces the bit rate of the $360^{\circ}$ video stream by dynamically selecting the viewport area and adjusting the quality of the viewport. The server side stores multiple adaptation sets related to the user's orientation, and matches them on the basis of the network state and viewport-position prediction. In this approach, each viewport that can be viewed is encoded and stored in multiple quality versions to meet different delivery requirements. To guarantee a smooth playback experience, mechanisms such as viewport prediction, synchronization with user head motion, and quality adjustment are also gaining attention. Corbillon et al. [43] proposed quality-focused regions, and set the video quality in these regions above other regions, thus reducing the huge bandwidth demand of $360^{\circ}$ video streams and achieving adaptive viewport video streams. Sreedhar et al. [44] compared ERP, $\mathrm{CMP}$, and pyramid projection, and proposed a differentiated quality approach that transmits the content in front of the projection at a relatively high resolution. Nguyen et al. [45] investigated the impact on $360^{\circ}$ viewport adaptive streaming of using response latency as an influencing factor. He et al. [46] proposed an FOV adaptive mechanism for the purpose of reducing bandwidth consumption. The length and size of the FoV segment were calculated on the basis of the measured network delay, so that the bandwidth could be efficiently used. Zhou et al. [47] proposed a new coding method to improve the problem of high loan consumption of $360^{\circ}$ video streams by coding more pixel information for the selected viewpoint direction to achieve the bit rate saving of the video.

However, in viewport-based streaming, a large amount of cache resources is required because of the need to adapt to the user's viewing direction according to network conditions, and the need for multiple versions of the content to be stored on the server side. When facing the service of live streaming, it is difficult to meet the resource-intensive coding in time because the user viewing position changes frequently, and a new high resolution is needed to replace viewport resources.

\subsubsection{Tile-Based Streaming}

In VR video streaming, video clips are generally divided into different clips on the basis of time sequences, and these video clips are then spatially divided into tiles of different sizes. The viewports are generally predicted in tile-based streaming to obtain the corresponding tiles to compose the desired viewports. Skupin et al. [48] encoded the tiles within the viewport on the basis of viewport adaptive streaming, namely, the tiles within the viewport that obtain different resolutions; by combining for different resolution tiles into the corresponding bit stream, resolution adaptation is achieved. Graf et al. [49] explored the properties of various tiles by studying $360^{\circ}$ videos, where each tile could be qualityadaptive projected according to different viewing areas. Results indicated significant bit rate saving compared to in the full- and partial-delivery strategies. Yu et al. in [50] divided an equirectangular video into many tiles, and where each horizontal tile was, it was assigned a sampling weight on the basis of its content. Bitrate allocation is optimized on the basis of sampling weights and bandwidth budget. The overlapping edges with two neighboring tiles are increased by blending at the edges of the overlapping tiles to overcome the probability of viewport loss. 
In some recent studies [51-53], the tiles within the viewport that the user needed to select were hierarchically represented as multiple types in order to overcome the problem of network changes and the randomness of head movements. Ozcinar et al. [51] optimized an adaptive omnidirectional video (ODV) streamer by means of an introduced visual-attention metric to achieve optimal streaming for each selected mode using a bit rate-allocation strategy that was assigned to tiles belonging to different regions. Xie et al. [54] constructed a probabilistic-model-based viewport prediction-error handling mechanism based on tile adaptive streaming to ensure the continuity of playback within the buffer. The authors also proposed to use the saliency model to further improve viewport adaptivity in the next phase of work. In [55], the authors improved the tile-based-stream coding method by comparing different tiles sizes and choosing the corresponding tiling scheme to achieve bit-stream saving.

Typically, tile-based streaming requires only a small number of content versions on the server side compared to viewport-related streaming, so it has lower storage and processing overhead. Viewports and adjacent tiles also reduce the bandwidth cost of streaming by using different resolutions. When there is an error in the viewport prediction, the video quality is significantly reduced because the tiles have different resolutions. Therefore, there is the huge problem of how to achieve a trade-off among the quality of the video stream, the viewport prediction error, and bandwidth efficiency.

\section{State of the Art of VR QoE}

In this section, we summarize the existing research on the main assessment methods and platforms for the QoE of VR users. The assessment methods for QoE are mainly divided into two main approaches: subjective and objective assessments. QoE usually represents the human perception of video quality; therefore, it needs to be quantified by the corresponding testing platforms and procedures.

\subsection{Subjective Quality Assessment}

Subjective evaluation is a challenging problem. The International Telecommunication Union (ITU) has proposed many experimental subjective video-quality evaluation methods. The results of subjective-evaluation experiments are mostly based on the quality level of user opinions and the final average results, where two metrics indicating the results, MOS [56] and DMOS [57], are widely used for subjective quality assessment. Some subjective assessment methods applicable to omnidirectional video are receiving attention. Huang et al. [58] proposed a study of omnidirectional images based on single-stimulus ACR. Perrin et al. [59] used a set of publicly available dataset images to compare the quality of HDR imaging using a new subjective assessment method for omnidirectional content aimed at omnidirectional representation. Shahid et al. [60], through subjective experiments, examined how the type of video content, camera motion position, and the number of moving targets may be factors that affect video in different network stopping states on user QoE. Azevedo et al. [61] argued that different people may explore content in different ways, but visual attention and saliency are the two main aspects that need to be considered during the subjective evaluation of $360^{\circ}$ video content. Van et al. [62] investigated the network disease problem by subjectively testing users' quality perception, experience, perception, and load problems. Anwar et al. [63] examined two key QoE influences, perceived quality and motion sickness, by collecting subjective experience datasets from 29 users on $96360^{\circ}$ viewings. The aim was to improve the motion-sickness aspects that occur during the viewing of $360^{\circ}$ videos. Kono et al. [64] used a subjective test of the repeated viewing of the same video sequence to quantify the relationship between video quality and presence through head movements and survey-question results. Other studies $[65,66]$ investigated the degree of influence of resolution, bit rate, quantization parameter $\mathrm{QP}$, and content characteristics on the perceived quality of $360^{\circ}$ videos and determined the main influencing factors for VR videos through subjective assessment tests. Schatz et al. [67] investigated the coding parameters, device type, perceived quality, and 
acceptability of 360 videos through subjective testing methods, and determined the number of influencing factors.

Unlike ordinary videos, $360^{\circ}$ videos are usually viewed using HMD devices for a better viewing experience. However, due to the limitations of human physiological characteristics, viewers can experience different degrees of adverse sensations such as strain and motion sickness when immersed in the content using HMDs. These undesirable sensations play a hindering role in enhancing QoE. Singla et al. [68] conducted a subjective quality-assessment test on tile-based $360^{\circ}$ video streaming to investigate the effect of video content, round-trip delay, and session duration on simulator disorders. In addition, the authors in $[69,70]$ proposed a modified absolute category rating (M-ACR) method by using different VR devices in order to analyze the halo phenomenon in $360^{\circ}$ videos under different bit rate and resolution conditions. Albert et al. [71] conducted a study of several key factors of the user (size of the eye-socket area, severity of degradation, degradation algorithm). A detailed investigation was conducted to understand how system latency affects the VR user experience on desktop and HMDs. Fernandes et al. [72] investigated the relationship between viewport size, VR sickness, and perceived quality using a subjective evaluation method. Steed et al. [73] examined a subjective VR device type, experience scenario, and the effect of the external environment. Huyen et al. [74] considered the effects of latency, quality changes, and interruptions on QoE, and the experimental results showed that the method had better prediction results and helped in the subjective evaluation of QoE to quantify the different quality of switching and interruptions. Yang et al. [75] proposed an objective assessment method applicable to panoramic videos and constructed a generic objective panoramic-video quality-assessment framework consisting of several quality factors and a fusion model. Table 3 summarizes the subjective quality assessment approaches.

Table 3. Subjective quality assessment approaches.

\begin{tabular}{|c|c|c|c|}
\hline Works & Important Influencing Factors & QoE Aspects & Years \\
\hline$[65,66]$ & $\begin{array}{c}\text { resolution, bit rate, quantization } \\
\text { parameter QP, content } \\
\text { characteristics }\end{array}$ & $\begin{array}{l}360 \text { degree video } \\
\text { perception quality }\end{array}$ & 2017 and 2018 \\
\hline [67] & $\begin{array}{l}\text { stalling patterns and variations in } \\
\text { encoding quality }\end{array}$ & $\begin{array}{l}\text { low perceived } \\
\text { resolution, low wearing } \\
\text { comfort, signs of } \\
\text { cybersickness, etc. }\end{array}$ & 2017 \\
\hline$[68-70]$ & $\begin{array}{l}\text { (videos sequences, resolution, } \\
\text { bandwidth, and network } \\
\text { round-trip delay) }\end{array}$ & simulator sickness & 2017, 2018 and 2019 \\
\hline$[71]$ & eye-tracking latency & $\begin{array}{c}\text { amount of foveation that } \\
\text { users }\end{array}$ & 2017 \\
\hline [72] & field-of-view modification & VR sickness & 2016 \\
\hline [73] & different types of system & $\begin{array}{l}\text { perception devices track } \\
\text { the hands and body }\end{array}$ & 2016 \\
\hline$[74]$ & $\begin{array}{l}\text { initial delay, quality variations and } \\
\text { interruptions }\end{array}$ & $\begin{array}{l}360 \text { degree video } \\
\text { perception quality }\end{array}$ & 2016 \\
\hline$[76]$ & $\begin{array}{l}\text { the saccadic suppression, foveal } \\
\text { vision, and contrast sensitivity of } \\
\text { the human visual system }\end{array}$ & $\begin{array}{l}360 \text { degree video } \\
\text { perception quality }\end{array}$ & 2020 \\
\hline
\end{tabular}

\subsection{Objective Quality Assessment}

At the present, in terms of the objective assessment of video, 2D flat video media has mature technical solutions. However, in the face of the spherical characteristics of $360^{\circ}$ video, the traditional flat objective quality-assessment method is directly used to analyze the panoramic video, and results cannot accurately reflect the quality of the video. Tran et al. [77] validated various traditional quality metrics and found that the traditional PSNR outperformed other pass metrics. Moller et al. [36] used data from subjective methods as a basis to predict the quality score (MOS) by objective data about the video, and results showed that some objective video quality-assessment methods advanced PSNR as a metric. Liu et al. [78] enhanced the validity of PSNR of spheres (S-PNSR) and perceptual 
PSNR (P-PSNR) for objective assessment of panoramic video. Objective and perceptual rate control (RC) formulas were developed to optimize these two objective metrics, enabling the best S-PSNR or P-PSNR results in panoramic-video coding. Yang et al. [76] focused on factors such as sweep suppression and the contrast sensitivity of the human visual system and conducted subjective experiments to investigate the perception of viewport adaption when quality changes occur. Zakharchenko et al. [79] proposed an objective evaluation method for weighted PSNR (W-PSNR) for $360^{\circ}$ video, normalizing the row-byrow addition of weighting coefficients derived from projection anisotropy. The results of this objective evaluation were confirmed by subjective visual tests.

SIM is used to define multifactor image distortion a quality-evaluation metric. Chen et al. [80] proposed a spherical SSIM (S-SSIM) metric for $360^{\circ}$ video by analyzing SSIM results to compare the similarity of the restored video and the original $360^{\circ}$ video. Tran et al. [77] performed a similar evaluation on 18 subjects. All considered objective metrics were highly correlated with the subjective results, and they attributed the source of distortion to changing the video-content format and transmitting the content over the network. Upenik et al. [81] analyzed the correlation between subjective quality and objective quality levels, and experimental results showed that in the existing objective metrics designed for $360^{\circ}$ videos did not reflect better subjective correlation than that of traditional objective evaluation metrics. The authors concluded that metrics developed for $360^{\circ}$ video content must undergo certain improvements to achieve better results. Lastly, Egan et al. [82] predicted biosensor-based QoE scores. Experimental results showed that electrical skin activity significantly contributed to the QoE score, while heart rate had a relatively small effect on the evaluation score. Table 4 summarizes the objective quality assessment approaches.

Table 4. Objective quality assessment approaches.

\begin{tabular}{|c|c|c|c|}
\hline Works & Metrics/Methods & Contribution & Years \\
\hline$[77]$ & PSNR & $\begin{array}{l}\text { Evaluation based on the correlation between objective } \\
\text { quality indicators and subjective quality. }\end{array}$ & 2017 \\
\hline$[75]$ & S-PSNR & $\begin{array}{l}\text { The objective evaluation and subjective relevance of this } \\
\text { work is higher than existing methods. }\end{array}$ & 2017 \\
\hline [78] & S-PSNR and P-PSNR & $\begin{array}{l}\text { The Rate control scheme is effective in improving the } \\
\text { S-PSNR and P-PSNR of panoramic video coding }\end{array}$ & 2018 \\
\hline [79] & weighted PSNR & $\begin{array}{l}\text { The accuracy and reliability of the proposed objective } \\
\text { quality estimation method have been verified, and it has a } \\
\text { good correlation with subjective quality estimation. }\end{array}$ & 2016 \\
\hline$[80]$ & S-SSIM & $\begin{array}{l}\text { S-SSIM outperforms state-of-the-art objective quality } \\
\text { assessment metrics in omnidirectional video quality } \\
\text { assessment. }\end{array}$ & 2018 \\
\hline [81] & $\begin{array}{l}\text { S-PSNR/WS- } \\
\text { PSNR/CPP- } \\
\text { PSNR/VIFP }\end{array}$ & $\begin{array}{l}\text { VIFP objective indicators provide the best performance } \\
\text { indicators. New algorithms are also needed to better } \\
\text { predict the perceived quality of omni-directional content. }\end{array}$ & 2017 \\
\hline$[82]$ & $\begin{array}{l}\text { Heart Rate and } \\
\text { ElectroDermal }\end{array}$ & $\begin{array}{l}\text { The first work to show the real relationship between the } \\
\text { EDA/HR combination and the QoE of users in an } \\
\text { immersive VR environment. }\end{array}$ & 2016 \\
\hline
\end{tabular}

\subsection{VR-Related QoE Evaluation Test Platform}

Due to the specificity of VR $360^{\circ}$ video experience, the design of subjective and objective assessment test platforms impacts the evaluation results. A number of studies designed test platforms according to their focuses.

Ahmadi et al. [83] proposed a test platform using an HMD as a display device regarding omnidirectional video and images. Upenik et al. [84] established a testbed for the subjective evaluation of VR, and to show the applicability of their testbed, the authors collected mean opinion scores (MOS) for $360^{\circ}$ images and videos of different quality levels through the testbed. Subjects' scores, orientation, and consumed time can be tracked from the testbed during each assessment session. Regal et al. [85] implemented a QoE testing platform for VR users using Unity, where testers were asked to fill in a questionnaire regarding their VR experience. During the test, the collected scores were stored in CSV files for result analysis. Singla et al. [69] constructed a QoE testbed and recruited 
28 subjects to evaluate six $360^{\circ}$ videos downloaded from YouTube, considering two commercial HMDs and two resolutions in the experiment. The disorders induced by viewing $360^{\circ}$ videos in HMDs were assessed by subjective evaluation. Bessa et al. [86] investigated the question of whether the experimental platform increased subjective QoE levels for $3 \mathrm{D}$ (stereoscopic) views compared to $2 \mathrm{D}$ views. By recruiting 63 participants, half of the participants watched the 2D version of the video, while the other half watched the 3D version. Singla et al. [70] developed a QoE testing platform, and recruited 28 subjects to evaluate six $360^{\circ}$ videos downloaded from YouTube, and two commercial HMDs and two resolutions were considered in the experiment. Illnesses induced by viewing $360^{\circ}$ videos in HMDs were assessed by subjective evaluation. Schatz et al. [87] considered VR-based training applications and investigated how the type of scene affected subjective scores and task performance. Hupont et al. [88] developed a QoE assessment procedure for games using HMDs and compared them with traditional displays regarding realism, and willingness to use for mobility; results indicated that HMDs all showed better QoE results. Han et al. [89] compared the assessment results of user QoE under different external conditions by both offline and online methods, which helped the system to make appropriate assessment choices. Gomes et al. [90] used a crowdsourcing approach to study the quality of experience (QoE) of VR self-driving cars through an Internet-based evaluation task to investigate system and human influences on the influence factor (IF) of the self-driving simulation. Midoglu et al. [91] constructed a measurement platform that correlates objective metrics with subjective user ratings for $360^{\circ}$ video streams. Simone et al. [92] analyze the quality of experience (QoE) of users by collecting subjective and objective data in VR interaction states. Although these studies shed some light on the testing and evaluation of QoE platforms for $360^{\circ}$ video, they are not involved in the optimization of $360^{\circ}$ video streams using QoE.

\section{QoE-Oriented Optimization for Virtual-Reality Video}

With the emergence of multimedia services and real-time application services, research on QoE intensified step by step, and the research on QoE optimization methods has been diversified. This section, we present the construction of a model about QoE based on VR video streams and the QoE-oriented VR optimization problem, and the QoE optimization strategies powered by applying machine-learning methods.

\subsection{VR QoE MODEL}

QoE models are built on the basis of the user's perceptual elements, and they are an important bridge in user and multimedia communication, both reflecting the user evaluation elements and act as a basis for later user optimization. Some of the most advanced QoE models [93-95] were examined, but they were all constructed in a 2D environment. Traditional QoE models for 2D video usually use video bit rate and network QoS parameters as input model input conditions.

To enable VR users to obtain a sense of immersive experience similar to real-world perception, VR video QoE tacitly considers a variety of factors related to VR characteristics such as stall speed, quality switching, and pauses. Several studies [96-98] selected corresponding QoE models for VR videos in order to better reflect the viewing experience of $360^{\circ}$ users. Kim et al. [96] established a relevant QoE prediction model by the fine-grained analysis of user perceptual motion characteristics and statistical content features affecting user motion perception from user's physiological characteristics, which aims to predict the degree of VR illness when watching VR videos to ensure a comfortable viewing experience. Experimental results showed that the correlation between the QoE model proposed in this paper for subjective disease scores reached $72 \%$. Yao et al. [97] studied and derived an HMD-based QoE model for $360^{\circ}$ video through an open-source $360^{\circ}$ video player for VR users, which explores diverse VR projection schemes. Experimental results showed better accuracy for the obtained QoE model with good scalability. The authors argued that more factors or more complex models are needed to improve modeling accuracy. Xie et al. [98] 
modeled this analytical process by studying the perceptual response to a mass change over a certain time period. Cross-validation of the data showed that the model exhibited very accurate mass estimates. Experimental results showed that both Pearson and Spearman's rank correlations exceeded 0.98. Yu et al. [99] used a psychological mapping model based on the Weber-Fechner law [100,101], combined with existing research $[102,103]$ assessment models and elements, and used the logarithmic function as the basic mapping relationship between QoE and VR video features to construct a relevant QoE model. Han et al. [104] examined the need to simultaneously guarantee the overall QoE maximization during the adaptive streaming of $360^{\circ}$ video in an environment with high network variability. The authors modeled this by using the QoE impact factors mentioned in the literature [105], where the QoE model is denoted as Equation (1), and Equation (2) represents the calculation process of the important parameter (impact of quality transformation) in the QoE model.

$$
\begin{gathered}
Q o E=q(R)-\mu \cdot t_{\text {stall }}-\lambda \cdot Q_{\text {switch }}-\omega \cdot t_{\text {startup }} \\
Q_{\text {switch }}=\sum_{\forall z \in \text { all zones } \forall c \in \text { all chunks }}\left|q\left(R^{\text {new }}\right)-q\left(R^{\text {old }}\right)\right|
\end{gathered}
$$

In the QoE model, in order to balance the impact of each major factor on the overall VR video QoE, they were assigned different coefficients. Where the bit rate of the video is denoted as the mapping function $\mathrm{q}(\mathrm{R})$, the damage effects of stall, quality switch, and startup time are denoted as $t_{\text {stall }}, Q_{\text {switch }}$ and $t_{\text {startup }}$, respectively. Experimental results showed that Q360AS has better QoE performance than that of PERCEIVE in streaming media.

Saxena et al. [106] studied virtual-reality headsets, modeled QoE for wireless-transmission intermittent behavior and user head-position prediction on the basis of $360^{\circ}$ different video streams, and proposed an MDP-based algorithm to accurately measure and optimize the cost and QoE per user. Roberto et al. [107] produced a platform for $360^{\circ}$ video visual-quality assessments. The platform provides access to multiple $360^{\circ}$ video viewport objective quality features and combines these features into QoE models that closely match subjective quality scores for a variety of different conditions. Hu et al. [108] used a QoE model for rate adaptation to maximize the utility of the QoE model under constraints by weighting the QoE parameters. Experiments show that VAS360 improves the user experience, and the quality of the viewport-adaptive solution was $23-45 \%$ better than that of the non-viewportadapt ive solution.

\subsection{QoE-Oriented VR Optimization Problem}

QoE currently represents the user's perception level well, so the optimization of VR video streams through QoE-oriented approaches is gaining increasing attention. They are also summarized in Table 5. Most current approaches [54,109] focus on optimizing QoE goals through specific heuristics. However, since user preferences differ, and usage scenarios vary greatly, using a single approach to optimize a specific QoE goal sometimes does not always yield good results. Therefore, it is important to choose the right optimization direction.

Existing $360^{\circ}$ video streaming systems mainly focus on optimizing specific qualityof-experience (QoE) goals through fixed heuristics $[54,109]$. However, users may have different preferences for QoE goals, and thus methods designed for specific scenarios cannot provide high QoE for all users. In addition, most existing methods rely on accurate predictions of future bandwidth and viewpoints, while dynamic changes in real scenarios significantly degrade the performance of these methods. A $360^{\circ}$ video viewport adaptive system driven by tile-based viewport QoE optimization is introduced by Xie et al. [54]. Figure 2 shows the architecture of the system. On the server side, a $360^{\circ}$ video is cropped by the video cropper with the encoder to generate an MPD document and stored on an HTTP server. A 360ProbDASH service is provided to the client. On the client side, we integrated additional modules in the DASH adaptation algorithm. (1) Direction Prediction: predicts the direction of user head movement; (2) Bandwidth Estimation: estimates the 
corresponding time-varying throughput on the basis of download duration; (3) QR Map: generates quality-rate $(\mathrm{QR})$ maps for all segments on the basis of attributes in the MPD; (4) Viewport Probability model: calculates the viewing probability of each tile with reference to the user's directional prediction error; (5) Target-buffer-based Rate Controller: controls the buffer to stay at the target level; (6) QoE-driven Optimizer: determines the optimal download segment involved in an HTTP GET request based on information from Modules 3-5. The system minimizes the expected spatial variation of quality distortion bricks and quality as the QoE optimization objectives under the constraint of the total transmitted bit rate. Experimental results showed that the method exhibited better results under the evaluation of some target metrics.

\section{Client Side}

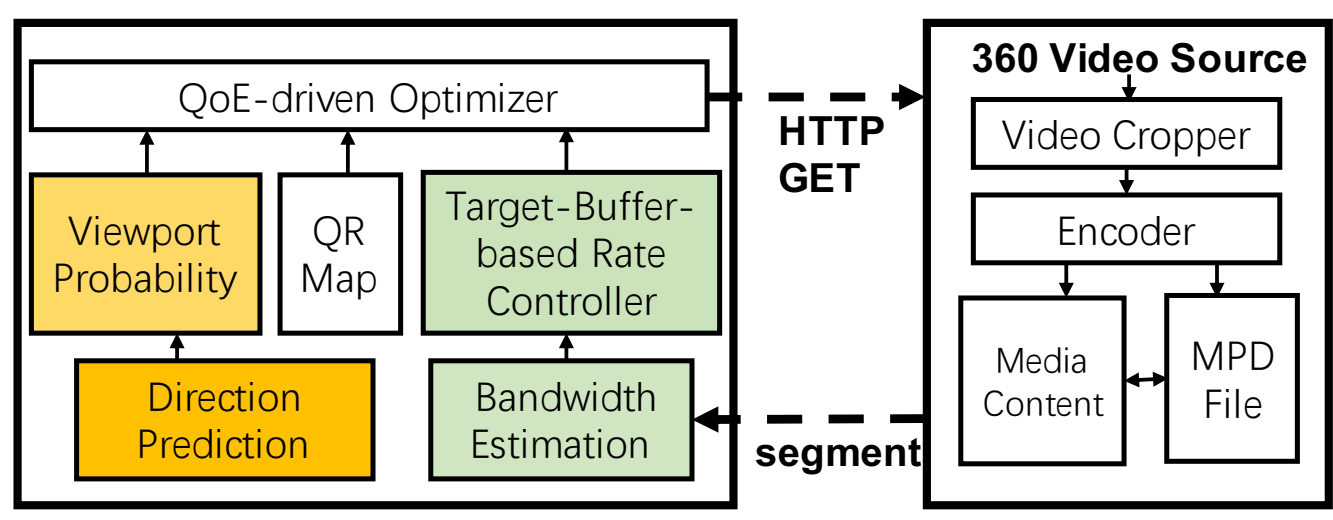

Figure 2. System architecture.

Hu et al. [110] proposed a novel $360^{\circ}$ video streaming algorithm based on user viewing behavior, improved the accuracy of viewport prediction by tile view maps constructed from real user line data, and optimized user QoE by saving limited bandwidth. Xie et al. [111] proposed a system for cross-user learning by studying a real VR user dataset and combining the obtained user viewing patterns. The optimization problem driven by tile rate allocation asking as QoE is optimized with the expectation of distortion under minimization. Wang et al. [112] designed a $360^{\circ}$ video self-adaptation scheme based on QoE optimization, and to ensure the maximization of multiuser (QoE) optimization and fairness, they jointly optimized the code rate delivery and cache decisions. The experimental results showed that this optimization method improves the cache hit rate and QoE performance compared to other methods. Zhang et al. [113] proposed EPASS360 to predict users' future views by mining patterns in other users' historical trajectories, set a QoE objective on the basis of prediction results with allocation strategy, and formulated a QoE optimization function to obtain the optimal rate allocation of tiles through a balanced selection. Experimental simulation results showed that EPASS360 was more competitive than advanced streaming performance, and QoE was improved in a variety of scenarios.

He et al. [114] designed a tile-based hierarchical coding framework for encoding spatial and temporal features of $360^{\circ}$ videos. It is also implemented in the client-side optimization process to optimize the QoE for the user. Figure 3 in this paper shows the comparison of the Rubiks system with existing optimized streaming algorithms. In this figure, YouTube [47] indicates: streaming all data for the entire $360^{\circ}$ frame to the client, FoV-only [115] indicates: streaming only the tiles predicted in the user's FoV. FoV+ [116] indicates: selecting the surrounding area on the basis of the estimated prediction error of the FoV. As the figure shows the optimization algorithm needs to consider the three important metrics of bandwidth saving, decoding speed and video quality at the same time. If excessive pursuit of video quality is considered as in Youtube, bandwidth and decoding speed are sacrificed. If bandwidth saving is needed to increase the decoding speed, the video quality is degraded, such as in FoV and FoV+. The Rubiks system wants to achieve the optimization goal by changing the encoding method of the tiles. 


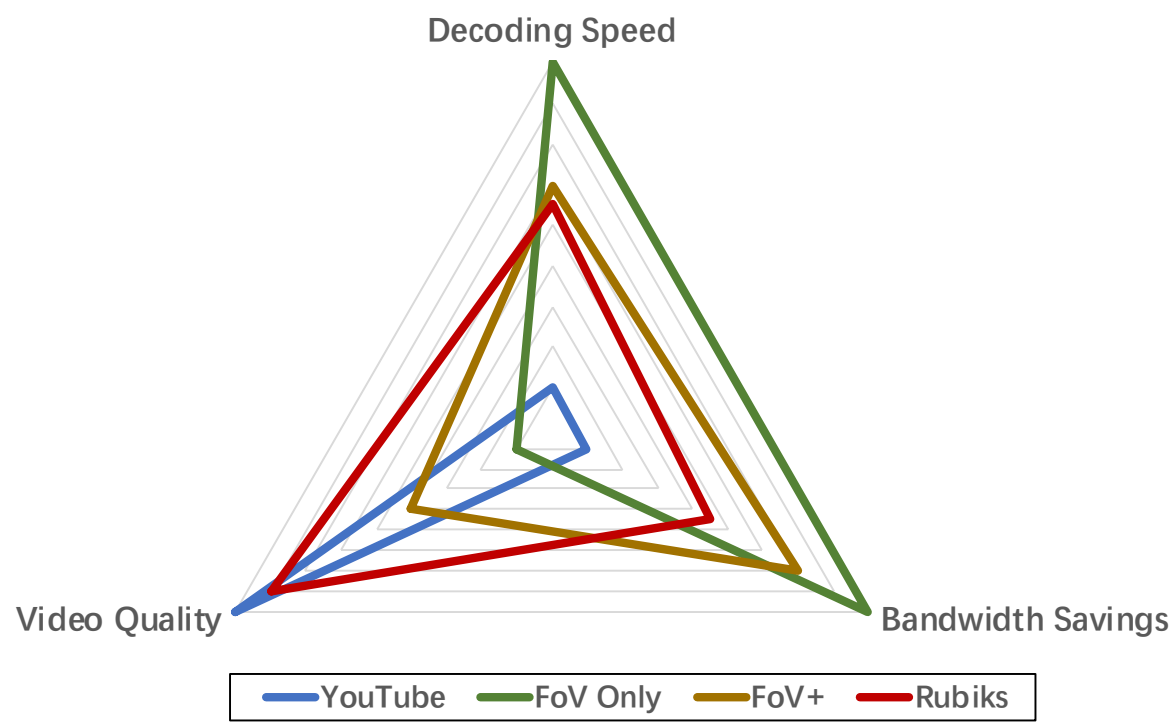

Figure 3. Design space of streaming algorithms.

Yu et al. [117] proposed a QoE-based video-adaptive method that combines QoE evaluation metrics and proposed an objective optimization-process function that adapts to the problem QoE; to ensure the flexibility of concealing, various preferences QoE parameters are adjustable. Experiments show that the method shows excellent results in different network environments. Perfecto et al. [118] proposed a method based on Lyapunov framework for the multicast problem maximizing the full network HD frame admission as a QoE optimization problem with the constraints of a low-latency and high-reliability optimization problem. Simulation results showed that content reuse with highly overlapping user clusters due to multicast reduces VR frame latency by $12 \%$.

Recently, several studies have found that the encoding of 360-degree video VR can have an impact on QoE. Appropriate settings of the video encoder driven by QoE aim to be optimal to achieve user QoE. Qian et al. [119] combined a subjective QoE model with an encoder parameter model to propose a QoE maximization problem with encoder adaptation as a constraint. Experimental results show that the proposed encoder adaptation scheme has a significant improvement on the user QoE. Tran et al. [65] investigate the effect of features such as encoding parameters and device type on the QoE aspect through principal observations and show that the video quality will be affected when the encoding level is reduced. Yang et al. [120] fully consider the encoding bit rate of each tile of VR video under ERP projection during resource allocation (RA), also considering the channel quality of each tile and user equipment (UE), and formulated this as a non-deterministic polynomial (NP)-hard problem, so a low-complexity approximate convex algorithm is proposed to solve it. The simulation results show that the overall viewer quality of experience (QoE) is significantly improved. Graf et al. [49] describe tile's implementation of bandwidth efficient adaptive streaming using modern video codecs such as HEVC/H.265 and VP9 to evaluate the quality of the viewport PSNR. Guan et al. [121] propose a video streaming system by considering the balance between perceptual quality and video coding efficiency; a variable-sized tile tiling coding scheme is proposed. The experimental results show that the perceptual QoE can be improved while reducing the bandwidth. 
Table 5. Summary of QoE-oriented VR optimization strategies.

\begin{tabular}{|c|c|c|}
\hline Strategy & Major Contributions & Objectives/Functionality \\
\hline Application-level optimization & $\begin{array}{l}\text { Flexibility QoE parameters } \\
\text { adjustable, Increase client } \\
\text { QoE-awareness }[54,117] .\end{array}$ & $\begin{array}{l}\text { Use contextual information to } \\
\text { improve the client's bit rate } \\
\text { selection strategy. }\end{array}$ \\
\hline $\begin{array}{l}\text { QoE-aware/driven adaptive } \\
\text { streaming based on user data }\end{array}$ & $\begin{array}{l}\text { Set QoE targets based on } \\
\text { forecast results and allocation } \\
\text { strategies }[110,111,113] \text {. }\end{array}$ & $\begin{array}{l}\text { Reduce content delivery latency } \\
\text { and improve network resource } \\
\text { utilization. }\end{array}$ \\
\hline $\begin{array}{l}\text { Video encoder with appropriate } \\
\text { settings to improve user QoE }\end{array}$ & $\begin{array}{l}\text { Tile-based layered coding } \\
\text { provides a balance between } \\
\text { quality and video coding } \\
\text { efficiency }[49,114,120,121] \text {. }\end{array}$ & $\begin{array}{l}\text { Improved tile coding method, } \\
\text { Optimize resource allocation } \\
\text { (RA), Increase the efficiency of } \\
\text { QoE and bandwidth usage. }\end{array}$ \\
\hline
\end{tabular}

\subsection{ML-Based Approaches Improve QoE}

The current development of machine-learning techniques is accompanied by their use in several fields. Some recent studies are on the use of machine-learning tools to evaluate these QoE models and participate in QoE performance optimization efforts. Costa Filho et al. [122] proposed a VR performance model applying techniques from both playback parameters and perceived QoE parameters through machine learning to predict the adaptive performance of VR systems while analyzing the impact of the network on VR streaming. The results showed good accuracy. Li et al. [123] considered data from human eye and head movements using a DRL model for the quality evaluation of $360^{\circ}$ videos. Yang et al. [75] considered individual pixels, regional superpixels, salient objects, and complete projections with inputs from multiple scales of the backpropagation (BP) algorithm. A quality assessment of a VR system (QAVR) metric was constructed. Li et al. [124] predicted the probability of viewing the possible viewports in the next phase and determined the extent of their impact on the expected QoE by CNN. Wu et al. [125] proposed a deepreinforcement-learning (DRL)-based approach ABR decision mechanism based on $360^{\circ}$ video streaming of tiles and implemented QoE, which can be adapted to multiple objective preference goals through a designed DQN model with preference encoder and customization. Ban et al. [126] used the mean field actor-critic (MFAC) algorithm to request viewing tiles with the aim of minimizing the bandwidth usage of the core network and maximizing the QoE with the user.

\section{Discussion: Challenges, Issues Future Directions}

In this section, we discuss the main challenges faced by QoE in VR video streaming applications and possible future research directions.

\subsection{Challenges and Impacts}

Currently, although most studies $[49,99,127,128]$ conducted various subjective and objective evaluations for $360^{\circ}$ videos, most evaluation methods are still directly selected from traditional video-evaluation criteria. There is a lack of uniform and standardized impact factors for $360^{\circ}$ videos. Standard evaluation methods are not yet finalized. This is a complex and challenging issue.

A study [129] with equivalent $\mathrm{HD}$ viewing experience for $360^{\circ}$ video required a viewport with $4 \mathrm{~K}$ by $4 \mathrm{~K}$ resolution at $60 \mathrm{fps}$, and a video requires $12 \mathrm{~K}$ resolution at $400 \mathrm{Mbps}$ bit rate. Different bit rates also result in a wide range of bandwidth requirements. With the further development of the VR experience level, various bandwidth and latency requirements are more stringent, and it is a challenging task to optimize the QoE metrics to ensure higher quality of experience (QoE) for $360^{\circ}$ video all the time.

\subsection{Issues for Future Directions}

\subsubsection{Research on User Personalization Modeling for VR Video Streaming QoE}

For VR video streams, the measure of VR video streams merit is quality of VR users' experience. At present, the main adopted measures are the PSNR and the utility function approach $[130,131]$. For VR video viewing through a head-mounted display, many factors 
such as the parameters related to the head-mounted display, and the rendering latency can affect the QoE experience of VR users. Therefore, according to a user's own institutional characteristics, adopting a more accurate mathematical model to describe the relationship of VR video streaming QoE and using the established applicable personalized QoE model as the goal of optimization is a problem worthy of in-depth investigation in future VR research.

\subsubsection{Trade-Off of QoE Based on MEC Solutions}

Currently, with the high speed of the fifth-generation communication technology, multiaccess edge computing (MEC) is considered [132] to have a driving role in VR development. Therefore, it is a very interesting research point to investigate the trade-off between the need to ensure high bandwidth and low latency in MEC-based VR systems and QoE optimization. QoE optimization of VR systems needs to consider reducing the cost of caching and computing while ensuring the user viewing experience. Balancing user QoE with cache and compute resource costs with MEC involvement is a future research direction.

\section{Conclusions}

With the spread of new VR devices and the increasing popularity of converged networks to provide VR applications, it is now a challenge to satisfy customers with a high-quality multimedia service experience by enabling users to perceive and evaluate the quality of experience of VR streaming services. To introduce readers to the latest and most widely used VR streaming technologies, the description of VR streaming services over the Internet focuses on viewport- and tile-based standards. Research shows that QoE assessment for VR requires an interdisciplinary perspective, so we chose to provide a comprehensive description of QoE influencing factors in terms of system, user, and context, with QoE multidimensional influencing factors generally serving as the basis for the entire QoE study. However, along with the continuous updating and development of VR services, the unification of QoE impact factors is not fully achieved.

QoE evaluation is based on platform measurements while considering the complex relationship between VR user characteristics and streaming system characteristics. We summarized the QoE assessment methods for VR and the related assessment platforms, which include two mainstream methods: subjective and objective assessment methods.

In QoE optimization, QoE-oriented elaboration adjustments are made, which in turn cycle to effectively improve QoE. On this basis, we investigated and discussed the construction of QoE models for VR users and QoE optimization for VR systems. The survey also extensively explored solutions for QoE evaluation and optimization using the emerging technology of machine learning. We also proposed and discussed future research needed in the following directions: QoE VR user-personalized QoE modeling studies and QoE trade-offs based on MEC solutions. The survey and current state of research provided in this paper could help readers to understand the direction of needed work.

Author Contributions: This work was mainly performed by J.R. (planning of the work, conceptualization, investigation, methodology, data curation, formal analysis, resources, software, visualization, and original draft preparation) and was completed with key contributions from D.X. (planning of the work, conceptualization, supervision, validation, manuscript review and editing and funding acquisition). Both authors have read and agreed to the published version of the manuscript.

Funding: This research received no external funding.

Conflicts of Interest: The authors declare no conflict of interest.

\section{Abbreviations}

The following abbreviations are used in this manuscript:

$\begin{array}{ll}\text { ACR } & \text { Absolute category rating } \\ \text { BP } & \text { Backpropagation } \\ \text { CMP } & \text { Cube map }\end{array}$




\begin{tabular}{|c|c|}
\hline CNN & Convolutional neural networks \\
\hline CPP-PSNR & Craster's parabolic projection peak signal-to-noise ratio \\
\hline CSV & Comma-separated values \\
\hline DASH & Dynamic adaptive streaming over HTTP \\
\hline DMOS & Difference mean opinion scores \\
\hline EM & Eye movement \\
\hline ERP & Equirectangular projection \\
\hline FoV & Field of view \\
\hline HD & High-definition video \\
\hline HEVC & High-efficiency video coding \\
\hline $\mathrm{HM}$ & Head movement \\
\hline HMD & Head-mounted display \\
\hline ITU & International Telecommunication Union \\
\hline MEC & Mobile edge computing \\
\hline ML & Machine learning \\
\hline M-ACR & Modified absolute category rating \\
\hline MOS & Mean Opinion Score \\
\hline M-SSIM & Mean structural similarity \\
\hline PSNR & Peak signal-to-noise ratio \\
\hline QEC & Quality emphasis center \\
\hline QP & Quantizer parameter \\
\hline S-PSNR & Spherical PSNR \\
\hline SRD & Systems reference document \\
\hline SSIM & Structural similarity index \\
\hline VIFP & Visual information fidelity in pixel domain \\
\hline VQA & Visual-quality assessment \\
\hline VQM & Video-quality metric \\
\hline VR & Virtual reality \\
\hline WS-PSNR & Weighted to Spherically uniform PSNR \\
\hline $2 \mathrm{D}$ & Two-dimensional \\
\hline $3 \mathrm{D}$ & Three-dimensional \\
\hline $4 \mathrm{~K}$ & $4 \mathrm{~K}$ resolution \\
\hline
\end{tabular}

\section{References}

1. Du, J.; Yu, F.R.; Lu, G.; Wang, J.; Jiang, J.; Chu, X. Mec-assisted immersive vr video streaming over terahertz wireless networks: A deep reinforcement learning approach. IEEE Internet Things J. 2020, 7, 9517-9529. [CrossRef]

2. Sukhmani, S.; Sadeghi, M.; Erol-Kantarci, M.; Saddik, A.E. Edge caching and computing in $5 \mathrm{~g}$ for mobile ar/vr and tactile internet. IEEE Multimed. 2018, 26, 21-30. [CrossRef]

3. ITU-T Recommendation ITU-T P. 10/g. 100 (11/2017). Vocabulary for Performance, Quality of Service and Quality of Experience. 2017. Available online: https:/ / www.itu.int/rec/T-REC-P.10 (accessed on 2 September 2021).

4. Avcibas, I.; Sankur, B.; Sayood, K. Statistical evaluation of image quality measures. J. Electron. Imaging 2002, 11, $206-223$.

5. Wang, Z.; Lu, L.; Bovik, A.C. Video quality assessment based on structural distortion measurement. Signal Process. Image Commun. 2004, 19, 121-132. [CrossRef]

6. Pinson, M.H.; Wolf, S. A new standardized method for objectively measuring video quality. IEEE Trans. Broadcast. 2004, 50, 312-322. [CrossRef]

7. ITU Telecommunication Standardization Sector. Objective perceptual multimedia video quality measurement in the presence of a full reference. ITU-T Recomm. J 2008, 247, 18.

8. Sousa, I.; Queluz, M.P.; Rodrigues, A. A survey on qoe-oriented wireless resources scheduling. J. Netw. Comput. Appl. 2020, 158, 102594. [CrossRef]

9. Zhao, T.; Liu, Q.; Chen, C.W. Qoe in video transmission: A user experience-driven strategy. IEEE Commun. Surv. Tutor. 2016, 19, 285-302. [CrossRef]

10. Juluri, P.; Tamarapalli, V.; Medhi, D. Measurement of quality of experience of video-on-demand services: A survey. IEEE Commun. Surv. Tutor. 2015, 18, 401-418. [CrossRef]

11. Yuan, H.; Hu, X.; Hou, J.; Wei, X.; Kwong, S. An ensemble rate adaptation framework for dynamic adaptive streaming over http. IEEE Trans. Broadcast. 2019, 66, 251-263. [CrossRef]

12. Barman, N.; Martini, M.G. Qoe modeling for http adaptive video streaming-A survey and open challenges. IEEE Access 2019, 7, 30831-30859. [CrossRef]

13. Chen, Y.; Wu, K.; Zhang, Q. From qos to qoe: A tutorial on video quality assessment. IEEE Commun. Surv. Tutor. 2014, 17, 1126-1165. [CrossRef] 
14. Husić, J.B.; Baraković, S.; Cero, E.; Slamnik, N.; Oćuz, M.; Dedović, A.; Zupčić, O. Quality of experience for unified communications: A survey. Int. J. Netw. Manag. 2020, 30, e2083.

15. Wang, Y.; Zhou, W.; Zhang, P. QoE Management in Wireless Networks; Springer: Berlin/Heidelberg, Germany, 2017.

16. Brunnström, K.; Beker, S.A.; Moor, K.D.; Dooms, A.; Egger, S.; Garcia, M.; Hossfeld, T.; Jumisko-Pyykkö, S.; Keimel, C.; Larabi, M.; et al. Qualinet White Paper on Definitions of Quality of Experience. 2013. Available online: https://hal.archives-ouvertes.fr/hal00977812/document (accessed on 2 September 2021).

17. ITUT Recommendation. Vocabulary for Performance and Quality of Service; International Telecommunications UnionRadiocommunication (ITU-T), RITP: Geneva, Switzerland, 2006.

18. Dobrian, F.; Sekar, V.; Awan, A.; Stoica, I.; Joseph, D.; Ganjam, A.; Zhan, J.; Zhang, H. Understanding the impact of video quality on user engagement. ACM SIGCOMM Comput. Commun. Rev. 2011, 41,362-373. [CrossRef]

19. Ghinea, G.; Thomas, J.P. Quality of perception: User quality of service in multimedia presentations. IEEE Trans. Multimed. 2005, 7, 786-789. [CrossRef]

20. Ickin, S.; Wac, K.; Fiedler, M.; Janowski, L.; Hong, J.H.; Dey, A.K. Factors influencing quality of experience of commonly used mobile applications. IEEE Commun. Mag. 2012, 50, 48-56. [CrossRef]

21. Balachandran, A.; Sekar, V.; Akella, A.; Seshan, S.; Stoica, I.; Zhang, H. A quest for an internet video quality-of-experience metric. In Proceedings of the 11th ACM Workshop on Hot Topics in Networks, Washington, WA, USA, 29-30 October 2012; pp. 97-102.

22. Huynh-Thu, Q.; Ghanbari, M. Temporal aspect of perceived quality in mobile video broadcasting. IEEE Trans. Broadcast. 2008, 54, 641-651. [CrossRef]

23. Han, B.; Zhang, X.; Qi, Y.; Gao, Y.; Yang, D. Qoe model based optimization for streaming media service considering equipment and environment factors. Wirel. Pers. Commun. 2012, 66, 595-612. [CrossRef]

24. Westerink, J.H.D.M.; Roufs, J.A.J. Subjective image quality as a function of viewing distance,resolution, and picture size. SMPTE J. 1989, 98, 113-119. [CrossRef]

25. Staelens, N.; Moens, S.; den Broeck, W.V.; Marien, I.; Vermeulen, B.; Lambert, P.; de Walle, R.V.; Demeester, P. Assessing quality of experience of iptv and video on demand services in real-life environments. IEEE Trans. Broadcast. 2010, 56, 458-466. [CrossRef]

26. Yamori, K.; Tanaka, Y. Relation between willingness to pay and guaranteed minimum bandwidth in multiple-priority service. In APCC/MDMC'04, Proceedings of the 2004 Joint Conference of the 10th Asia-Pacific Conference on Communications and the 5th International Symposium on Multi-Dimensional Mobile Communications Proceeding Beijing, China, 29 August-1 September 2004; IEEE: Washington, DC, USA, 2004; Volume 1, pp. 113-117.

27. Sackl, A.; Schatz, R.; Raake, A. More than i ever wanted or just good enough? user expectations and subjective quality perception in the context of networked multimedia services. Qual. User Exp. 2017, 2, 3. [CrossRef]

28. Sackl, A.; Schatz, R. Got what you want? modeling expectations to enhance web qoe prediction. In Proceedings of the 2014 Sixth International Workshop on Quality of Multimedia Experience (QoMEX), Singapore, 18-20 September 2014; pp. 57-58.

29. Sackl, A.; Schatz, R. Evaluating the influence of expectations, price and content selection on video quality perception. In Proceedings of the 2014 Sixth International Workshop on Quality of Multimedia Experience (QoMEX), Singapore, 18-20 September 2014; pp. 93-98.

30. Laghari, K.U.R.; Connelly, K. Toward total quality of experience: A qoe model in a communication ecosystem. IEEE Commun. Mag. 2012, 50, 58-65. [CrossRef]

31. Owsley, C.; Sekuler, R.; Siemsen, D. Contrast sensitivity throughout adulthood. Vis. Res. 1983, 23, 689-699. [CrossRef]

32. El-Nasr, M.S.; Yan, S. Visual attention in 3d video games. In Proceedings of the 2006 ACM SIGCHI International Conference on Advances in Computer Entertainment Technology, Hollywood, CA, USA, 14-16 June 2006; p. 22.

33. Saleme, E.B.; Covaci, A.; Assres, G.; Comsa, I.; Trestian, R.; Santos, C.A.S.; Ghinea, G. The influence of human factors on $360^{\circ}$ mulsemedia qoe. Int. J. Hum.-Comput. Stud. 2021, 146, 102550. [CrossRef]

34. Orero, P.; Remael, A.; Cintas, J.D. Media for All: Subtitling for the Deaf, Audio Description, and Sign Language; Rodopi: Amsterdam, The Netherlands, 2007.

35. Wechsung, I.; Schulz, M.; Engelbrecht, K.P.; Niemann, J.; Möller, S. All users are (not) equal-the influence of user characteristics on perceived quality, modality choice and performance. In Proceedings of the Paralinguistic Information and Its Integration in Spoken Dialogue Systems Workshop; Springer: Berlin/Heidelberg, Germany, 2011; pp. 175-186.

36. Möller, S.; Raake, A. Quality of Experience: Advanced Concepts, Applications and Methods; Springer: Berlin/Heidelberg, Germany, 2014.

37. Silvia, P.J. Interest-The curious emotion. Curr. Dir. Psychol. Sci. 2008, 17, 57-60. [CrossRef]

38. O'Brien, H.L.; Toms, E.G. What is user engagement? a conceptual framework for defining user engagement with technology. J. Am. Soc. Inf. Sci. Technol. 2008, 59, 938-955. [CrossRef]

39. Kortum, P.; Sullivan, M. The effect of content desirability on subjective video quality ratings. Hum. Factors 2010, 52, 105-118. [CrossRef] [PubMed]

40. Palhais, J.; Cruz, R.S.; Nunes, M.S. Quality of experience assessment in internet tv. In International Conference on Mobile Networks and Management; Springer: Berlin/Heidelberg, Germany, 2011; pp. 261-274.

41. Zhu, Y.; Heynderickx, I.; Redi, J.A. Understanding the role of social context and user factors in video quality of experience. Comput. Hum. Behav. 2015, 49, 412-426. [CrossRef]

42. Afzal, S.; Chen, J.; Ramakrishnan, K.K. Characterization of 360-degree videos. In Proceedings of the Workshop on Virtual Reality and Augmented Reality Network, Los Angeles, CA, USA, 25 August 2017; pp. 1-6. 
43. Corbillon, X.; Simon, G.; Devlic, A.; Chakareski, J. Viewport-adaptive navigable 360-degree video delivery. In Proceedings of the 2017 IEEE International Conference on Communications (ICC), Paris, France, 21-25 May 2017; pp. 1-7.

44. Sreedhar, K.K.; Aminlou, A.; Hannuksela, M.M.; Gabbouj, M. Viewport-adaptive encoding and streaming of 360-degree video for virtual reality applications. In Proceedings of the 2016 IEEE International Symposium on Multimedia (ISM), San Jose, CA, USA, 11-13 December 2016; pp. 583-586.

45. Nguyen, D.V.; Tran, H.T.T.; Thang, T.C. Impact of delays on 360-degree video communications. In Proceedings of the 2017 TRON Symposium (TRONSHOW), Tokyo, Japan, 13-15 December 2017; pp.1-6.

46. He, D.; Westphal, C.; Garcia-Luna-Aceves, J.J. Joint rate and fov adaptation in immersive video streaming. In Proceedings of the 2018 Morning Workshop on Virtual Reality and Augmented Reality Network, Budapest, Hungary, 24 August 2018 ; pp. 27-32.

47. Zhou, C.; Li, Z.; Liu, Y. A measurement study of oculus 360 degree video streaming. In Proceedings of the 8 th ACM on Multimedia Systems Conference, Taipei, Taiwan, 20-23 June 2017; pp. 27-37.

48. Skupin, R.; Sanchez, Y.; Hellge, C.; Schierl, T. Tile based hevc video for head mounted displays. In Proceedings of the 2016 IEEE International Symposium on Multimedia (ISM), San Jose, CA, USA, 11-13 December 2016; pp. 399-400.

49. Graf, M.; Timmerer, C.; Mueller, C. Towards bandwidth efficient adaptive streaming of omnidirectional video over http: Design, implementation, and evaluation. In Proceedings of the 8th ACM on Multimedia Systems Conference, Taipei, Taiwan, 20-23 June 2017; pp. 261-271.

50. Yu, M.; Lakshman, H.; Girod, B. Content adaptive representations of omnidirectional videos for cinematic virtual reality. In Proceedings of the 3rd International Workshop on Immersive Media Experiences, Brisbane, Australia, 30 October 2015; pp. 1-6.

51. Ozcinar, C.; Cabrera, J.; Smolic, A. Visual attention-aware omnidirectional video streaming using optimal tiles for virtual reality. IEEE J. Emerg. Sel. Top. Circuits Syst. 2019, 9, 217-230. [CrossRef]

52. Nguyen, D.V.; Tran, H.T.T.; Pham, A.T.; Thang, T.C. An optimal tile-based approach for viewport-adaptive 360-degree video streaming. IEEE J. Emerg. Sel. Top. Circuits Syst. 2019, 9, 29-42. [CrossRef]

53. Hosseini, M.; Swaminathan, V. Adaptive 360 vr video streaming: Divide and conquer. In Proceedings of the 2016 IEEE International Symposium on Multimedia (ISM), San Jose, CA, USA, 11-13 December 2016; pp. 107-110.

54. Xie, L.; Xu, Z.; Ban, Y.; Zhang, X.; Guo, Z. 360probdash: Improving qoe of 360 video streaming using tile-based http adaptive streaming. In Proceedings of the 25th ACM International Conference on Multimedia, New York, NY, USA, 23-27 October 2017; pp. 315-323.

55. Zare, A.; Aminlou, A.; Hannuksela, M.M.; Gabbouj, M. Hevc-compliant tile-based streaming of panoramic video for virtual reality applications. In Proceedings of the 24th ACM international conference on Multimedia, Amsterdam, The Netherlands, 15-19 October 2016; pp. 601-605.

56. Tan, T.K.; Weerakkody, R.; Mrak, M.; Ramzan, N.; Baroncini, V.; Ohm, J.; Sullivan, G.J. Video quality evaluation methodology and verification testing of hevc compression performance. IEEE Trans. Circuits Syst. Video Technol. 2015, 26, 76-90. [CrossRef]

57. Seshadrinathan, K.; Soundararajan, R.; Bovik, A.C.; Cormack, L.K. Study of subjective and objective quality assessment of video. IEEE Trans. Image Process. 2010, 19, 1427-1441. [CrossRef]

58. Huang, M.; Shen, Q.; Ma, Z.; Bovik, A.C.; Gupta, P.; Zhou, R.; Cao, X. Modeling the perceptual quality of immersive images rendered on head mounted displays: Resolution and compression. IEEE Trans. Image Process. 2018, 27, 6039-6050. [CrossRef]

59. Perrin, A.F.; Bist, C.; Cozot, R.; Ebrahimi, T. Measuring quality of omnidirectional high dynamic range content. In Applications of Digital Image Processing XL; International Society for Optics and Photonics: Bellingham, WA, USA, 2017; Volume 10396, p. 1039613.

60. Anwar, M.S.; Wang, J.; Ahmad, S.; Ullah, A.; Khan, W.; Fei, Z. Evaluating the factors affecting qoe of 360-degree videos and cybersickness levels predictions in virtual reality. Electronics 2020, 9, 1530. [CrossRef]

61. de A Azevedo, R.G.; Birkbeck, N.; De Simone, F.; Janatra, I.; Adsumilli, B.; Frossard, P. Visual distortions in 360-degree videos. arXiv 2019, arXiv:1901.01848.

62. Van Kasteren, A.; Brunnström, K.; Hedlund, J.; Snijders, C. Quality of experience assessment of 360-degree video. Electron. Imaging 2020, 2020, 91. [CrossRef]

63. Anwar, M.S.; Wang, J.; Khan, W.; Ullah, A.; Ahmad, S.; Fei, Z. Subjective qoe of 360-degree virtual reality videos and machine learning predictions. IEEE Access 2020, 8, 148084-148099. [CrossRef]

64. Kono, T.; Hayashi, T. Subjective qoe assessment method for $360^{\circ}$ videos. IEICE Commun. Express 2020, 10, 174-178. [CrossRef]

65. Tran, H.T.T.; Ngoc, N.P.; Pham, C.T.; Jung, Y.J.; Thang, T.C. A subjective study on qoe of 360 video for vr communication. In Proceedings of the 2017 IEEE 19th International Workshop on Multimedia Signal Processing (MMSP), London-Luton, UK, 16-18 October 2017; pp. 1-6.

66. Younus, M.U. Analysis of the impact of different parameter settings on wireless sensor network lifetime. Int. J. Adv. Comput. Sci. Appl. 2018, 9, 16-21.

67. Schatz, R.; Sackl, A.; Timmerer, C.; Gardlo, B. Towards subjective quality of experience assessment for omnidirectional video streaming. In Proceedings of the 2017 Ninth International Conference on Quality of Multimedia Experience (QoMEX), Erfurt, Germany, 29 May-2 June 2017; pp. 1-6.

68. Singla, A.; Göring, S.; Raake, A.; Meixner, B.; Koenen, R.; ; Buchholz, T. Subjective quality evaluation of tile-based streaming for omnidirectional videos. In Proceedings of the 10th ACM Multimedia Systems Conference, Amherst, MA, USA, 18-21 June 2019; pp. 232-242. 
69. Singla, A.; Fremerey, S.; Robitza, W.; Raake, A. Measuring and comparing qoe and simulator sickness of omnidirectional videos in different head mounted displays. In Proceedings of the 2017 Ninth International Conference on Quality of Multimedia Experience (QoMEX), Erfurt, Germany, 29 May-2 June 2017; pp. 1-6.

70. Singla, A.; Fremerey, S.; Robitza, W.; Lebreton, P.; Raake, A. Comparison of subjective quality evaluation for hevc encoded omnidirectional videos at different bit-rates for uhd and fhd resolution. In Proceedings of the on Thematic Workshops of ACM Multimedia 2017, Mountain View, CA, USA, 23-27 October 2017; pp. 511-519.

71. Albert, R.; Patney, A.; Luebke, D.; Kim, J. Latency requirements for foveated rendering in virtual reality. ACM Trans. Appl. Percept. (TAP) 2017, 14, 1-13. [CrossRef]

72. Fernandes, A.S.; Feiner, S.K. Combating vr sickness through subtle dynamic field-of-view modification. In Proceedings of the 2016 IEEE Symposium on 3D User Interfaces (3DUI), Greenville, SC, USA, 19-20 March 2016; pp. 201-210.

73. Steed, A.; Frlston, S.; Lopez, M.M.; Drummond, J.; Pan, Y.; ; Swapp, D. An 'in the wild' experiment on presence and embodiment using consumer virtual reality equipment. IEEE Trans. Vis. Comput. Graph. 2016, 22, 1406-1414. [CrossRef]

74. Tran, H.T.T.; Ngoc, N.P.; Pham, A.T.; Thang, T.C. A multi-factor qoe model for adaptive streaming over mobile networks. In Proceedings of the 2016 IEEE Globecom Workshops (GC Wkshps), Washington, WA, USA, 4-8 December 2016 ; pp. 1-6.

75. Yang, S.; Zhao, J.; Jiang, T.; Wang, J.; Rahim, T.; Zhang, B.; Xu, Z.; Fei, Z. An objective assessment method based on multi-level factors for panoramic videos. In Proceedings of the 2017 IEEE Visual Communications and Image Processing (VCIP), Petersburg, FL, USA, 10-13 December 2017; pp. 1-4.

76. Yang, M.; Zou, W.; Song, J.; Yang, F. Enhancing qoe for viewport-adaptive 360-degree video streaming: Perception analysis and implementation. IEEE Multimed. 2020. [CrossRef]

77. Tran, H.T.T.; Ngoc, N.P.; Bui, C.M.; Pham, M.H.; Thang, T.C. An evaluation of quality metrics for 360 videos. In Proceedings of the 2017 Ninth International Conference on Ubiquitous and Future Networks (ICUFN), Milan, Italy, 4-7 July $2017 ;$ pp. 7-11.

78. Liu, Y.; Yang, L.; Xu, M.; Wang, Z. Rate control schemes for panoramic video coding. J. Vis. Commun. Image Represent. 2018, 53, 76-85. [CrossRef]

79. Zakharchenko, V.; Choi, K.P.; Park, J.H. Quality metric for spherical panoramic video. In Optics and Photonics for Information Processing X; International Society for Optics and Photonics: Bellingham, WA, USA, 2016; Volume 9970, p. 99700C.

80. Chen, S.; Zhang, Y.; Li, Y.; Chen, Z.; Wang, Z. Spherical structural similarity index for objective omnidirectional video quality assessment. In Proceedings of the 2018 IEEE International Conference on Multimedia and Expo (ICME), San Diego, CA, USA, 23-27 July 2018; pp. 1-6.

81. Upenik, E.; Rerabek, M.; Ebrahimi, T. On the performance of objective metrics for omnidirectional visual content. In Proceedings of the 2017 Ninth International Conference on Quality of Multimedia Experience (QoMEX), Erfurt, Germany, 29 May-2 June 2017; pp. $1-6$.

82. Egan, D.; Brennan, S.; Barrett, J.; Qiao, Y.; Timmerer, C.; Murray, N. An evaluation of heart rate and electrodermal activity as an objective qoe evaluation method for immersive virtual reality environments. In Proceedings of the 2016 Eighth International Conference on Quality of Multimedia Experience (QoMEX), Lisbon, Portugal, 6-8 June 2016; pp. 1-6.

83. Ahmadi, H.; Eltobgy, O.; Hefeeda, M. Adaptive multicast streaming of virtual reality content to mobile users. In Proceedings of the Thematic Workshops of ACM Multimedia 2017, Mountain View, CA, USA, 23-27 October 2017; pp. 170-178.

84. Upenik, E.; Řeřábek, M.; Ebrahimi, T. Testbed for subjective evaluation of omnidirectional visual content. In Proceedings of the 2016 Picture Coding Symposium (PCS), Nuremberg, Germany, 4-7 December 2016; pp. 1-5.

85. Regal, G.; Schatz, R.; Schrammel, J.; Suette, S. Vrate: A unity3d asset for integrating subjective assessment questionnaires in virtual environments. In Proceedings of the 2018 Tenth International Conference on Quality of Multimedia Experience (QoMEX), Sardinia, Italy, 29-31 May 2018; pp. 1-3.

86. Bessa, M.; Melo, M.; Narciso, D.; Barbosa, L.; Vasconcelos-Raposo, J. Does 3d 360 video enhance user's vr experience? An evaluation study. In Proceedings of the XVII International Conference on Human Computer Interaction, Salamanca, Spain, 13-16 September 2016; pp. 1-4.

87. Schatz, R.; Regal, G.; Schwarz, S.; Suettc, S.; Kempf, M. Assessing the qoe impact of 3d rendering style in the context of vr-based training. In Proceedings of the 2018 Tenth International Conference on Quality of Multimedia Experience (QoMEX), Sardinia, Italy, 29-31 May 2018; pp. 1-6.

88. Hupont, I.; Gracia, J.; Sanagustin, L.; Gracia, M.A. How do new visual immersive systems influence gaming qoe? a use case of serious gaming with oculus rift. In Proceedings of the 2015 Seventh International Workshop on Quality of Multimedia Experience (QoMEX), Messinia, Greece, 26-29 May 2015; pp. 1-6.

89. Han, Y.; Yu, C.; Li, D.; Zhang, J.; Lai, Y. Accuracy analysis on $360^{\circ}$ virtual reality video quality assessment methods. In Proceedings of the 2020 IEEE/ ACM 13th International Conference on Utility and Cloud Computing (UCC), Leicester, UK, 7-10 December 2020; pp. 414-419.

90. Gomes, G.D.; Flynn, R.; Murray, N. A crowdsourcing-based qoe evaluation of an immersive vr autonomous driving experience. In Proceedings of the 2021 13th International Conference on Quality of Multimedia Experience (QoMEX), Montreal, QC, Canada, 14-17 June 2021; pp. 25-30.

91. Midoglu, C.; Klausen, M.; Alay, Ö; Yazidi, A.; Haugerud, H.; Griwodz, C. Poster: Qoe-based analysis of real-time adaptive 360-degree video streaming. In Proceedings of the 2019 on Wireless of the Students, by the Students, and for the Students Workshop, Los Cabos, Mexico, 21 October 2019; p. 13. 
92. Simone, F.D.; Li, J.; Debarba, H.G.; Ali, A.E.; Gunkel, S.N.B.; Cesar, P. Watching videos together in social virtual reality: An experimental study on user's qoe. In Proceedings of the 2019 IEEE Conference on Virtual Reality and 3D User Interfaces (VR), Osaka, Japan, 23-27 March 2019; pp. 890-891.

93. Mao, H.; Netravali, R.; Alizadeh, M. Neural adaptive video streaming with pensieve. In Proceedings of the Conference of the ACM Special Interest Group on Data Communication, Los Angeles, CA, USA 21-25 August 2017; pp. 197-210.

94. Petrangeli, S.; Famaey, J.; Claeys, M.; Latré, S.; Turck, F.D. Qoe-driven rate adaptation heuristic for fair adaptive video streaming. ACM Trans. Multimed. Comput. Commun. Appl. (TOMM) 2015, 12, 1-24. [CrossRef]

95. Yin, X.; Jindal, A.; Sekar, V.; Sinopoli, B. A control-theoretic approach for dynamic adaptive video streaming over http. In Proceedings of the 2015 ACM Conference on Special Interest Group on Data Communication, London, UK, 17-21 August 2015 pp. 325-338.

96. Kim, J.; Kim, W.; Ahn, S.; Kim, J.; Lee, S. Virtual reality sickness predictor: Analysis of visual-vestibular conflict and vr contents. In Proceedings of the 2018 Tenth International Conference on Quality of Multimedia Experience (QoMEX), Sardinia, Italy, 29 May-1 June 2018; pp. 1-6.

97. Yao, S.; Fan, C.; Hsu, C. Towards quality-of-experience models for watching 360 videos in head-mounted virtual reality. In Proceedings of the 2019 Eleventh International Conference on Quality of Multimedia Experience (QoMEX), Berlin, Germany, 5-7 June 2019; pp. 1-3.

98. Xie, S.; Xu, Y.; Qian, Q.; Shen, Q.; Ma, Z.; Zhang, W. Modeling the perceptual impact of viewport adaptation for immersive video. In Proceedings of the 2018 IEEE International Symposium on Circuits and Systems (ISCAS), Florence, Italy, 27-30 May 2018; pp. 1-5.

99. Yu, M.; Lakshman, H.; Girod, B. A framework to evaluate omnidirectional video coding schemes. In Proceedings of the 2015 IEEE International Symposium on Mixed and Augmented Reality, Fukuoka, Japan, 29 September-3 October 2015; pp. 31-36.

100. Fiedler, M.; Hossfeld, T.; Tran-Gia, P. A generic quantitative relationship between quality of experience and quality of service. IEEE Netw. 2010, 24, 36-41. [CrossRef]

101. Reichl, P.; Egger, S.; Schatz, R.; D'Alconzo, A. The logarithmic nature of qoe and the role of the weber-fechner law in qoe assessment. In Proceedings of the 2010 IEEE International Conference on Communications, Enschede, The Netherlands, 7-9 July 2010; pp. 1-5.

102. Cermak, G.; Pinson, M.; Wolf, S. The relationship among video quality, screen resolution, and bit rate. IEEE Trans. Broadcast. 2011, 57, 258-262. [CrossRef]

103. Belmudez, B.; Moller, S. An approach for modeling the effects of video resolution and size on the perceived visual quality. In Proceedings of the 2011 IEEE International Symposium on Multimedia, Dana Point, CA, USA, 5-7 December 2011; pp.464-469.

104. Han, Y.; Ma, Y.; Liao, Y.; Muntean, G. Qoe oriented adaptive streaming method for $360^{\circ}$ virtual reality videos. In Proceedings of the 2019 IEEE SmartWorld, Ubiquitous Intelligence \& Computing, Advanced \& Trusted Computing, Scalable Computing \& Communications, Cloud \& Big Data Computing, Internet of People and Smart City Innovation (SmartWorld/SCALCOM/UIC/ATC/CBDCom/IOP/SCI), Leicester, UK, 19-23 August 2019; pp. 1655-1659.

105. Piamrat, K.; Viho, C.; Bonnin, J.M.; Ksentini, A. Quality of experience measurements for video streaming over wireless networks. In Proceedings of the 2009 Sixth International Conference on Information Technology: New Generations, Las Vegas, NV, USA, 27-29 April 2009; pp.1184-1189.

106. Saxena, A.; Subramanyam, S.; Cesar, P.; van der Mei, R.; van den, B.J. Efficient, qoe aware delivery of $360^{\circ}$ videos on vr headsets over mobile links. In Proceedings of the 13th EAI International Conference on Performance Evaluation Methodologies and Tools, Tsukuba, Japan, 18-20 May 2020; pp. 156-163.

107. Roberto, G.D.; Birkbeck, N.; Janatra, I.; Adsumilli, B.; Frossard, P. Multi-feature 360 video quality estimation. IEEE Open J. Circuits Syst. 2021, 2, 338-349.

108. Hu, Y.; Liu, Y.; Wang, Y. Vas360: Qoe-driven viewport adaptive streaming for 360 video. In Proceedings of the 2019 IEEE International Conference on Multimedia \& Expo Workshops (ICMEW), Shanghai, China, 8-12. July 2019; pp. 324-329.

109. Huang, W.; Ding, L.; Wei, H.Y.; Hwang, J.; Xu, Y.; Zhang, W. Qoe-oriented resource allocation for 360-degree video transmission over heterogeneous networks. arXiv 2018, arXiv:1803.07789.

110. Zai, M.; Chen, J.; Wu, D.; Zhou, Y.; Wang, Y.; Dai, H. Tvg-streaming: Learning user behaviors for qoe-optimized 360-degree video streaming. IEEE Trans. Circuits Syst. Video Technol. 2020. [CrossRef]

111. Xie, L.; Zhang, X.; Guo, Z. Cls: A cross-user learning based system for improving qoe in 360-degree video adaptive streaming. In Proceedings of the 26th ACM International Conference on Multimedia, Seoul, Korea, 22-26 October 2018; pp. 564-572.

112. Wang, S.; Tan, X.; Li, S.; Xu, X.; Yang, J.; Zheng, Q. A qoe-based $360^{\circ}$ video adaptive bitrate delivery and caching scheme for c-ran. In Proceedings of the 2020 16th International Conference on Mobility, Sensing and Networking (MSN), Tokyo, Japan, 17-19 December 2020; pp. 49-56.

113. Zhang, Y.; Guan, Y.; Bian, K.; Liu, Y.; Tuo, H.; Song, L.; Li, X. Epass360: Qoe-aware 360-degree video streaming over mobile devices. IEEE Trans. Mob. Comput. 2020, 20, 2338-2353. [CrossRef]

114. He, J.; Qureshi, M.A.; Qiu, L.; Li, J.; Li, F.; Han, L. Rubiks: Practical 360-degree streaming for smartphones. In Proceedings of the 16th Annual International Conference on Mobile Systems, Applications, and Services, Munich, Germany, 10-15 June 2018; pp. 482-494. 
115. Qian, F.; Ji, L.; Han, B.; Gopalakrishnan, V. Optimizing 360 video delivery over cellular networks. In Proceedings of the 5th Workshop on All Things Cellular: Operations, Applications and Challenges, New York City, NY, USA,3-7 October 2016; pp. 1-6.

116. Bao, Y.; Wu, H.; Zhang, T.; Ramli, A.A.; Liu, X. Shooting a moving target: Motion-prediction-based transmission for 360-degree videos. In Proceedings of the 2016 IEEE International Conference on Big Data (Big Data), Washington, WA, USA, 5-8 December 2016; pp. 1161-1170.

117. Yu, L.; Tillo, T.; Xiao, J. Qoe-driven dynamic adaptive video streaming strategy with future information. IEEE Trans. Broadcast. 2017, 63, 523-534. [CrossRef]

118. Perfecto, C.; Elbamby, M.S.; Ser, J.D.; Bennis, M. Taming the latency in multi-user vr $360^{\circ}$ : A qoe-aware deep learning-aided multicast framework. IEEE Trans. Commun. 2020, 68, 2491-2508. [CrossRef]

119. Qian, L.; Cheng, Z.; Fang, Z.; Ding, L.; Yang, F.; Huang, W. A qoe-driven encoder adaptation scheme for multi-user video streaming in wireless networks. IEEE Trans. Broadcast. 2016, 63, 20-31. [CrossRef]

120. Yang, J.; Luo, J.; Meng, D.; Hwang, J.N. Qoe-driven resource allocation optimized for uplink delivery of delay-sensitive vr video over cellular network. IEEE Access 2019, 7, 60672-60683. [CrossRef]

121. Guan, Y.; Zheng, C.; Zhang, X.; Guo, Z.; Jiang, J. Pano: Optimizing 360 video streaming with a better understanding of quality perception. In Proceedings of the ACM Special Interest Group on Data Communication, Beijing, China, 19-23 August 2019; pp. 394-407.

122. da Costa Filho, R.I.T.; Luizelli, M.C.; Vega, M.T.; van der Hooft, J.; Petrangeli, S.; Wauters, T.; Turck, F.D.; Gaspary, L.P. Predicting the performance of virtual reality video streaming in mobile networks. In Proceedings of the 9th ACM Multimedia Systems Conference, Amsterdam, The Netherlands, 12-15 June 2018; pp. 270-283.

123. Li, C.; Xu, M.; Du, X.; Wang, Z. Bridge the gap between vqa and human behavior on omnidirectional video: A large-scale dataset and a deep learning model. In Proceedings of the 26th ACM International Conference on Multimedia, Seoul, Korea, 22-26 October 2018; pp. 932-940.

124. Li, C.; Xu, M.; Jiang, L.; Zhang, S.; Tao, X. Viewport proposal cnn for 360deg video quality assessment. In Proceedings of the IEEE/CVF Conference on Computer Vision and Pattern Recognition, Long Beach, CA, USA, 16-20 June 2019; pp. 10177-10186.

125. Wu, C.; Wang, Z.; Sun, L. Paas: A preference-aware deep reinforcement learning approach for $360^{\circ}$ video streaming. In Proceedings of the 31st ACM Workshop on Network and Operating Systems Support for Digital Audio and Video, Istanbul, Turkey, 28 September-1 October 2021; pp. 34-41.

126. Ban, Y.; Zhang, Y.; Zhang, H.; Zhang, X.; Guo, Z. Ma360: Multi-agent deep reinforcement learning based live 360-degree video streaming on edge. In Proceedings of the 2020 IEEE International Conference on Multimedia and Expo (ICME), London, UK, 6-10 July 2020; pp. 1-6.

127. Gaddam, V.R.; Riegler, M.; Eg, R.; Griwodz, C.; Halvorsen, P. Tiling in interactive panoramic video: Approaches and evaluation. IEEE Trans. Multimed. 2016, 18, 1819-1831. [CrossRef]

128. Zhang, B.; Zhao, J.; Yang, S.; Zhang, Y.; Wang, J.; Fei, Z. Subjective and objective quality assessment of panoramic videos in virtual reality environments. In Proceedings of the 2017 IEEE International Conference on Multimedia \& Expo Workshops (ICMEW), Hong Kong,China, 10-14 July 2017; pp.163-168

129. Mangiante, S.; Klas, G.; Navon, A.; GuanHua, Z.; Ran, J.; Silva, M.D. Vr is on the edge: How to deliver 360 videos in mobile networks. In Proceedings of the Workshop on Virtual Reality and Augmented Reality Network, Los Angeles, CA, USA, 25 August 2017; pp. 30-35.

130. Cicco, L.D.; Mascolo, S. An adaptive video streaming control system: Modeling, validation, and performance evaluation. IEEE/ACM Trans. Netw. 2013, 22, 526-539. [CrossRef]

131. Xu, Y.; Zhou, Y.; Chiu, D.M. Analytical qoe models for bit-rate switching in dynamic adaptive streaming systems. IEEE Trans. Mob. Comput. 2014, 13, 2734-2748. [CrossRef]

132. Hsu, C. Mec-assisted fov-aware and qoe-driven adaptive $360^{\circ}$ video streaming for virtual reality. In Proceedings of the 202016 th International Conference on Mobility, Sensing and Networking (MSN), Tokyo, Japan, 17-19 December 2020; pp. 291-298. 